\title{
Energy harvesting for wireless autonomous sensor systems
}

Rob van Schaijk

Imec/Holst Centre

High Tech Campus 31, 5605 KN Eindhoven, the Netherlands

\section{INTRODUCTION}

The continuously decreasing power consumption of silicon-based electronics has enabled a broad range of battery-powered handheld, wearable and even implantable devices. A variety of wireless devices having power consumption spanning six orders of magnitude are listed in Table I, with their typical autonomy.

Table I. Selected battery-operated systems

\begin{tabular}{|l|c|c|}
\hline \multirow{2}{*}{} & \multicolumn{2}{|c|}{ Device Type } \\
\cline { 2 - 3 } & $\begin{array}{c}\text { Power } \\
\text { Consumption }\end{array}$ & $\begin{array}{c}\text { Energy } \\
\text { Autonomy }\end{array}$ \\
\hline Smartphone & $1 \mathrm{~W}$ & 5 hours \\
\hline MP3 player & $50 \mathrm{~mW}$ & 15 hours \\
\hline Hearing Aid Sensor & $1 \mathrm{~mW}$ & 5 days \\
\hline $\begin{array}{l}\text { Wireless Node } \\
\text { Cardiac Pacemaker }\end{array}$ & $50 \mu \mathrm{WW}$ & Lifetime \\
\hline Quartz watch & $5 \mu \mathrm{W}$ & 5 years \\
\hline
\end{tabular}

All these devices need a compact, low-cost and lightweight energy source, which allows the desired portability and energy autonomy. Today, batteries represent the dominant energy source for the devices listed in Table I and alike. In spite of the fact that energy density of batteries has increased by a factor of 3 over the past 15 years, their presence in many cases has a large impact on, or even dominate, the overall size and operational cost of devices. For this reason, alternative solutions to batteries are the subject of worldwide extensive research and development. One possibility is to replace them with energy storage systems featuring higher energy density, e.g., miniaturized fuel cells [1]. A second possibility consists of providing the required energy to the device in a wireless mode. This solution, already used for RFID tag, can be extended to more power hungry devices, but it requires dedicated transmission infrastructures. A third possibility is harvesting energy from the ambient by using, for example, vibration/motion energy, thermal energy, light or RF radiation.

The power consumption of a wireless sensor node (WSN) has been estimated by various authors with quoted values between 1 and $20 \mu \mathrm{W}$ in recent works
[2-3]. As schematically illustrated in Figure 1, the components of a typical WSN include micropower module, sensor/actuator, front-end processing unit, digital signal processor and radio. The power consumption strongly depends on the complexity of the sensed physical effect and the number of times it has to be transmitted per second. Practical implementation of a sensor node shows that $90 \mu \mathrm{W}$ is enough to drive a pulse oxymeter sensor, to process data and to transmit them at an interval of 15 seconds [4]. Whereas $10 \mu \mathrm{W}$ turns out to be sufficient to measure and transmit temperature readings every 5 seconds [5]. The value of $100 \mu \mathrm{W}$ reported in Table $\mathrm{I}$ is therefore representative of relatively complex sensor nodes, for systems operating at a relatively high data rate.

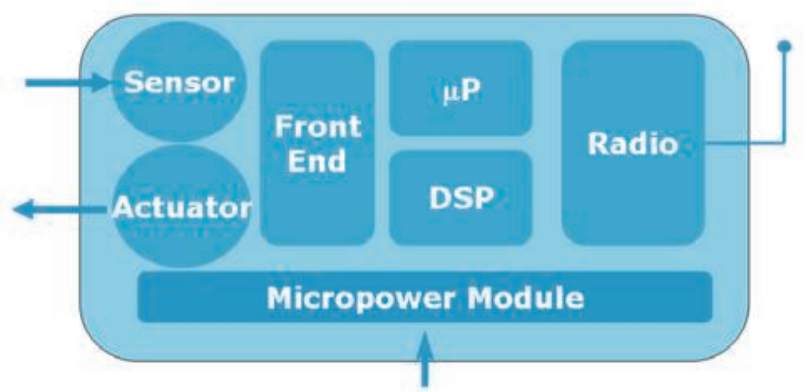

Figure 1. Schematic representation of the components of a typical WSN.

Wireless sensor networks are made up of a large number of small, low-cost sensor nodes working in collaboration to collect data and transmit them to a base station via a wireless link. They are finding increasingly extensive applications in body area networks and health monitoring of machinery, industrial and civil structures.

These networks are intended in many cases to operate for a period of years. Because of the large number of devices and their small size, replacing depleted batteries is unpractical or simply not feasible. Enlarging the size of the battery to ensure energy autonomy throughout the lifetime of the system would increase system size and cost beyond what is tolerable. The combination of an energy harvester with a small-sized rechargeable battery (or with another energy storage system like a thin-film rechargeable battery or a supercapacitor) is the best approach to realize energy autonomy of the network throughout the entire lifetime. 
For instance, if the power consumption of a sensor node is approximately $100 \mu \mathrm{W}$, the lifetime of a primary battery is expected to be only a few months [5]. In comparison, the combination of a rechargeable battery and an energy harvester with a power output of $100 \mu \mathrm{W}$ is able to ensure energy autonomy for the whole lifetime.

It is worth noting that abolishing the energy storage system altogether is not an option in most cases. As shown in Figure 2, the peak currents needed by the wireless transceiver during transmit and receive operation go beyond what is achievable by using the harvester alone. Furthermore, buffering is also desired to ensure continuous operation during times with no power generated. Depending on the application, the energy storage system can be a battery or a supercapacitor.

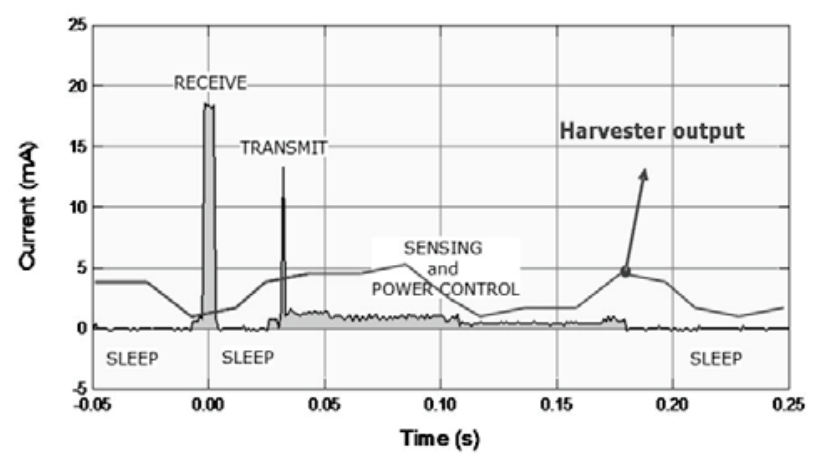

Figure 2. A typical scenario for the power consumption of a sensor node. Since the consumption does not equally match the harvester output, an energy buffer and power management IC in between are necessary.

For successful implementation of harvesting devices, three major factors have been identified:

a) The price of the harvesting device has to be reduced relative to the price of a complete WSN;

b) The power delivered by the harvester should be sufficient to support the desired functionality of WSN;

c) Power consumption of WSN has to be minimized via the use of ultra low power optimization techniques and technology breakthroughs.

Cost consideration clearly depends on the chosen application. For example, in case of infrastructure monitoring for predictive maintenance, the price of an individual WSN can be relatively high, because the accumulated cost reduction is far greater than the initial investment. Condition monitoring of infrastructures is also exactly the field where the first energy harvesters have appeared on the market.

However, for most other applications, current harvesting technologies are still far too expensive. A possible route to cheaper harvesting devices is using MEMS technology for manufacturing. The devices can thus be fabricated on a wafer basis in a batch mode, thereby greatly reducing the cost. Nevertheless, reducing the size of a harvesting device not only lowers the cost but also the power output.

As to the power delivered by the different energy harvesting methods, Table II summarizes the data presented in a previous publication. It suggests that energy harvesters can supply approximately $10 \mu \mathrm{W}$ $-1 \mathrm{~mW}$.

\begin{tabular}{|c|c|c|}
\hline Source & $\begin{array}{l}\text { Source } \\
\text { power }\end{array}$ & $\begin{array}{l}\text { Harvested } \\
\text { power }\end{array}$ \\
\hline $\begin{array}{l}\text { Ambient light } \\
\text { Indoor } \\
\text { Outdoor }\end{array}$ & $\begin{array}{l}0.1 \mathrm{~mW} / \mathrm{cm}^{2} \\
100 \mathrm{~mW} / \mathrm{cm}^{2}\end{array}$ & $\begin{array}{l}10 \mu \mathrm{W} / \mathrm{cm}^{2} \\
10 \mathrm{~mW} / \mathrm{cm}^{2}\end{array}$ \\
\hline $\begin{array}{l}\text { Vibration/motion } \\
\text { Human }\end{array}$ & $\begin{array}{l}0.5 \mathrm{~m} @ 1 \mathrm{~Hz} \\
1 \mathrm{~m} / \mathrm{s}^{2} @ 50 \mathrm{~Hz}\end{array}$ & $4 \mu \mathrm{W} / \mathrm{cm}^{2}$ \\
\hline $\begin{array}{l}\text { Vibration/motion } \\
\text { Industrial }\end{array}$ & $\begin{array}{l}1 \mathrm{~m} @ 5 \mathrm{~Hz} \\
10 \\
\mathrm{~m} / \mathrm{s}^{2} @ 1 \mathrm{kHz}\end{array}$ & $100 \mu \mathrm{W} / \mathrm{cm}^{2}$ \\
\hline $\begin{array}{l}\text { Thermal Energy } \\
\text { Human } \\
\text { Industrial }\end{array}$ & $\begin{array}{l}20 \mathrm{~mW} / \mathrm{cm}^{2} \\
100 \mathrm{~mW} / \mathrm{cm}^{2}\end{array}$ & $\begin{array}{l}30 \mu \mathrm{W} / \mathrm{cm}^{2} \\
1-10 \mathrm{~mW} / \mathrm{cm}^{2}\end{array}$ \\
\hline $\begin{array}{l}\text { RF } \\
\text { Cell phone }\end{array}$ & $0.3 \mu \mathrm{W} / \mathrm{cm}^{2}$ & $0.1 \mu \mathrm{W} / \mathrm{cm}^{2}$ \\
\hline
\end{tabular}

It is expected that energy harvesters will improve in the coming years, especially those based on vibration and temperature difference. The use of micromachining will result in cheaper devices with higher power output per unit volume. In this paper, we focus on vibration and thermal harvesting. We discuss their basic principles and their implementaition using micromachining technology.

\section{Piezoelectric Vibration energy harVesting}

\section{A. Introduction}

For converting motion or vibration into electricity, the established transduction mechanisms include electrostatic, piezoelectric or electromagnetic. In electrostatic transducers, the distance or overlap between the two electrodes of a polarized capacitor varies due to the displacement or vibration of one movable electrode. This motion induces a voltage change across the capacitor, and results in a current flow in an external circuit. In piezoelectric transducers, vibration or movement causes the 
deformation of a piezoelectric capacitor thereby generating a voltage. In electromagnetic transducers, the displacement of a magnetic mass with respect to a coil produces a change in the magnetic flux. This leads to an AC voltage appearing across the coil.

Vibration harvesters are by far the most widely investigated in the literature. Fine-machined versions are the earliest emerging commercial devices while micro-electro-mechanical system (MEMS, or sometimes also referred to as micro-systems technology, MST) versions are far less mature for the moment [7-8]. At the micrometer scale, electromagnetic is not sufficiently scalable and therefore, most efforts are dedicated to the piezoelectric and electrostatic transduction methods. Interesting applications are condition monitoring in machineries and automobiles, like tire pressure monitoring systems, for which a large market volume is foreseen. The results shown in this section are attained on a MEMS-based vibration energy harvester with aluminum nitride (AIN) as piezoelectric material.

\section{B. Principle}

A simple electromechanical model of a piezoelectric vibrator has been proposed by Williams and Yates [9] and adopted by Mitcheson et al. [10]. In this model, the physical behavior of a general piezoelectric generator is modeled as a damped mechanical vibrator consisting of a generic massspring system with a driving force. According to that model, the maximum power $P_{\max }$, which is dissipated in the damper and thus converted into electrical or mechanical energy, can be obtained at the resonance frequency $\left(\omega_{0}\right)$ as

$$
P_{\max }=\frac{1}{2} m \frac{Q_{t o t}}{\omega_{0}} \alpha_{0}^{2}
$$

where $\boldsymbol{Q}_{\text {tot }}$ is the total (electrical and mechanical) quality factor and $\alpha_{0}$ is the input acceleration. However, Roundy et.al. has elaborated that the maximum power that can be extracted from the piezoelectric vibration harvester is [11-12]:

$$
P_{\text {max }}=\frac{1}{4}\left(\frac{e_{31}^{2}}{\varepsilon_{0} \varepsilon_{33}^{T}}\right)\left(\frac{1-v_{b}}{Y_{b}}\right)\left(\frac{m}{\omega_{0}}\right) Q_{t o t}^{2} \alpha_{0}^{2}
$$

where $e_{31}$ is the piezoelectric constant, $\varepsilon_{33}^{T}$ is the relative permittivity of the piezoelectric element under constant stress, $\varepsilon_{0}$ is the permittivity of the free space (equal to $8.85 \times 10^{-12} \mathrm{~F} / \mathrm{m}$ ). For a thin film piezoelectric layer, Young's modulus $\left(Y_{\mathrm{b}}\right)$ and Poisson's ratio $\left(v_{b}\right)$ of the substrate material, instead of those of the piezoelectric film itself, are to be taken into consideration. This simplified model gives a good indication of the maximum power generation obtained with piezoelectric energy harvesters.

\section{Fabrication and packaging}

As shown in Figure 3, the device consists of a cantilever structure with a piezoelectric capacitor on top of the beam and an attached mass. The piezoelectric capacitor is formed by consecutive deposition, lithography and etching steps on a platinum bottom electrode, an AIN piezoelectric layer and an aluminum top electrode. The silicon mass and beam are formed by subsequent front and backside etching, where both beam thickness and uniformity are controlled by using SOI wafers [13]. The most commonly used piezoelectric material is lead zirconate titanate (PZT), but in our devices AIN is used due to its easier sputtering process and comparable electromechanical coupling coefficients.

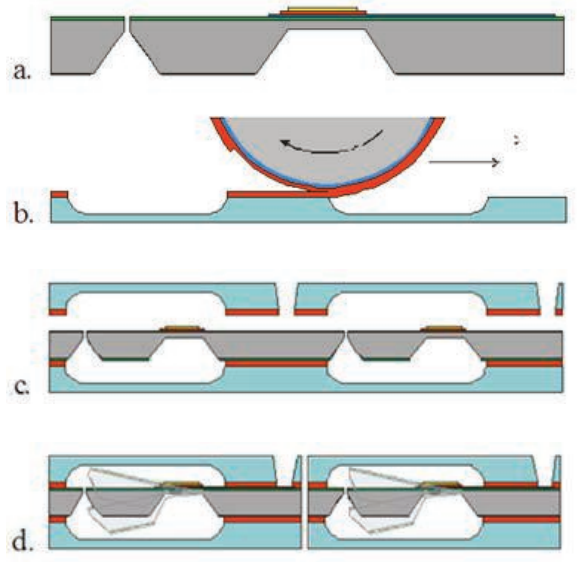

Figure 3 Schematic fabrication process flow: (a) The AIN piezoelectric capacitor is located on top of the beam; (b) The SU-8 bonding layer is applied with a wafer-scale roller-coating process on the glass wafers; (c) The two glass wafers and the silicon wafer are vacuum bonded in two consecutive bonding steps; (d) After dicing, single devices are obtained with the movable mass and beam in the vacuum cavity.

An important requirement of MEMS-based energy harvesters is the robustness of the device and for this a good package is needed. The package prevents excessive mass displacements and protects the device from external influences. As shown later, vacuum is essential to minimize losses due to air damping. As illustrated in Figure 3, the device wafer is encapsulated with a top and bottom glass wafer. In both wafers, cavities are etched and the bonding layer is applied with a wafer-scale rollercoating process. The main advantage of the roller- 
coating process is the selective deposition of adhesive on the desired areas. After vacuum bonding, singlated devices are obtained by dicing. Photographs of a 6-inch device wafer and a typical vacuum packaged device are shown in Figure 4.
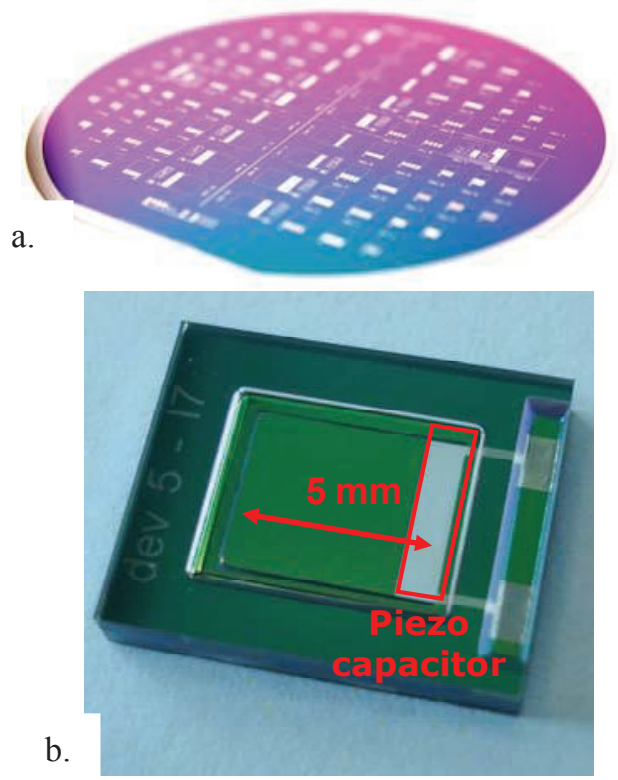

Figure 4 (a) 6" wafer with devices with different dimensions; (b) device with glass cover and contact openings. The mass and the piezeoelectric capacitor can be clearly observed.

\section{Device characterization}

As mentioned before, the vacuum is required to minimize air damping and maximize the power output. The effect of air damping is exhibited in Figure 5 for two different acceleration levels. A significant gain, by two orders of magnitude, can be achieved in power output. The small $\left(<1 \mathrm{~cm}^{3}\right)$ MEMS-based energy harvester described in this paper delivers a power output up to $100 \mu \mathrm{W}$ for $1.0 \mathrm{~g}$ acceleration. In Figure 6, power output for different accelerations are shown for a device with resonance frequency of $572 \mathrm{~Hz}$ and quality factor of $\sim 500$. Ongoing research effort is concentrated on further improvement of the hermeticity of package aimed to prevent leakage. The leakage is found to be dependent on device size and in general, power loss by $50 \%$ is observed over a period of six months. In Figure 7 , the measured resonance curves are obtained with different time intervals after initial vacuum packaging, for an acceleration of $0.32 \mathrm{~g}$.

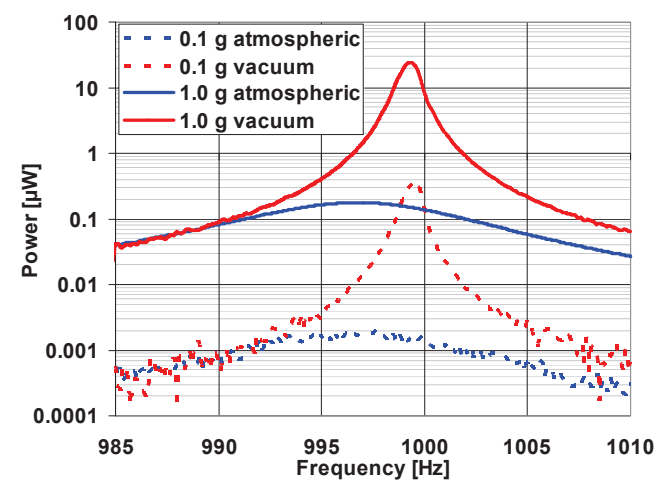

Figure 5. Resonance curves of packaged device at atmospheric pressure and vacuum for two different acceleration levels.

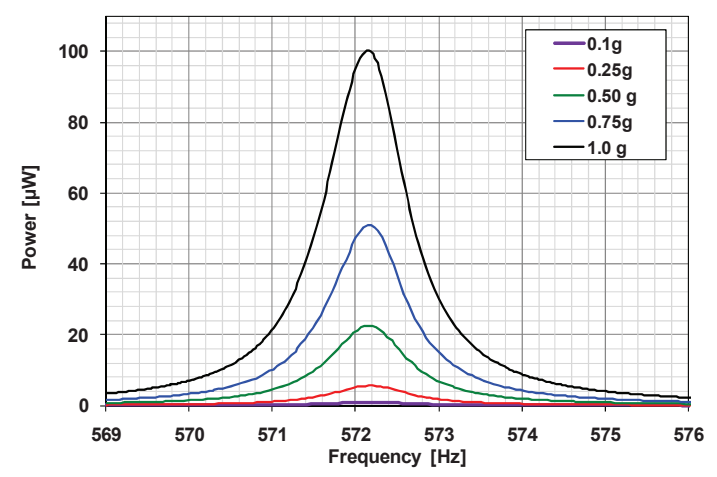

Figure 6. Power output of a vacuum packaged device with 100 $\mu \mathrm{W}$ for $1.0 \mathrm{~g}$ acceleration.

The power output decreases at all frequencies due to increased parasitic damping as air leaks in over time. The leakage into the package through the SU-8 bonding layer is a diffusion process driven by the pressure difference between the inside and outside of the package. The diffusion can be reduced by using a thinner SU-8 layer or by increasing the width of the bonding. A second option is to make two or more ring-shaped SU-8 seals instead of a single one. Simulations show that this will supress the diffusion into the package effectively.

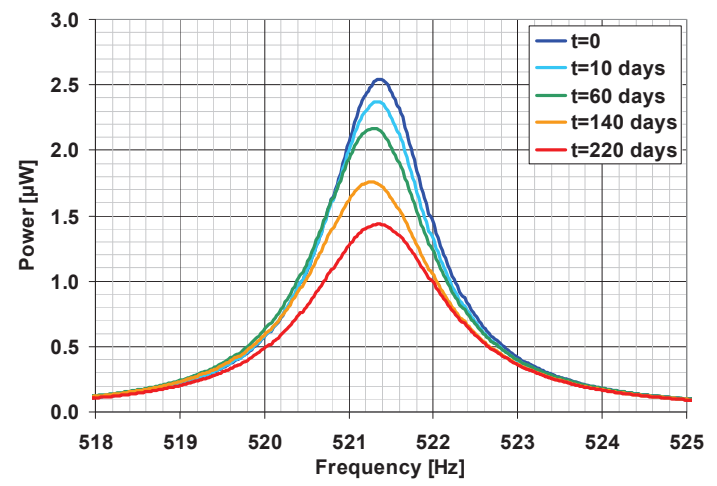

Figure 7. Reduction of power output for different periods after manufacturing due to leak into the package 
A third option can be implemented by replacing the polymer SU-8 bond with a metallic eutectic bond, which will not suffer from diffusion and is known to be hermetic [14].

\section{Thermoelectric EnERgy HARVESting}

\section{A. Introduction}

The thermoelectric energy harvesting is based on the Seebeck effect, which was discovered about two centuries ago and named after its discoverer [15]. This physical phenomenon is related to the generation of an electrical potential difference due to the presence of a temperature difference across a metal or semiconductor material. Its physical origin can be attributed to several effects, among which the temperature-driven shift of Fermi level, the thermal diffusion of charge carriers and the phonon drag effect are the most important ones. In general, the Seebeck coefficient, the generated voltage per unit temperature difference, is inversely proportional to the charge carrier concentration. This fact indicates that the magnitude of the Seebeck coefficient is usually moderate for metals while tunable for semiconductor materials by doping. Dependent on the type of dopant, the Seebeck coefficient can be either positive or negative.

The most widely used device based on the Seebeck effect is the thermocouple, which is usually formed by two materials with Seebeck coefficients in opposite signs. Its primary application is temperature sensing for measurement and control. When a number of thermocouples are connected electrically in series and thermally in parallel, a thermopile is then realized. With the schematic general configuration shown in Figure 8, a thermopile delivers an open-circuit voltage output across the two open terminals when subject to a temperature difference. This voltage output $V_{o c}$ can be expressed as

$$
V_{o c}=n\left(\alpha_{p}-\alpha_{n}\right) \Delta T
$$

where $n$ is the total number of thermocouples, $\alpha_{p}$ and $\alpha_{n}$ are Seebeck coefficients for p-type and ntype materials respectively, $\Delta T$ is the temperature difference. The generated voltage can be used to drive an electrical current through an external load connected to this thermoelectric generator (TEG). Thus, electrical power is harvested from the heat flow through the TEG.

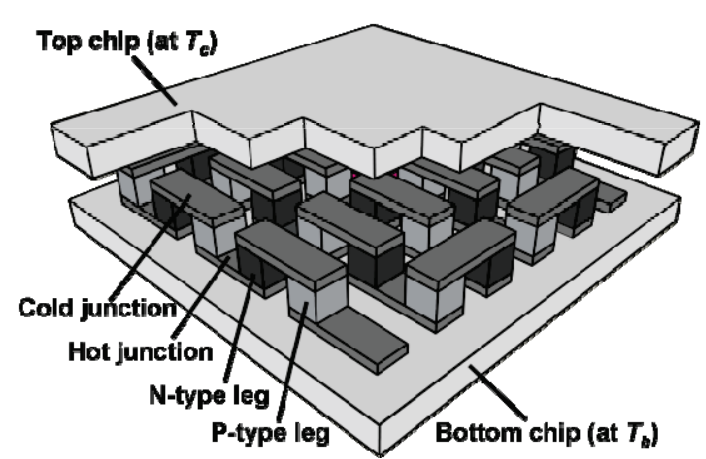

Figure 8. Schematic configuration of a general thermoelectric generator.

\section{B. Fabrication and characterization}

When used for energy harvesting, commercial thermopiles are less favorable for several reasons. Mostly assembled by manual craftsmanship, the thermocouples usually have a relatively large geometry and thus a moderate thermal resistance. This fact gives rise to a small fraction of an overall temperature difference falling across the thermopile in the presence of serial parasitic thermal resistors. Moreover, each individual thermopile only contains a limited number of thermocouples, leading to an inadequate output performance.

Micromachining technology enables the fabrication of a large number of miniature thermocouples with geometry, configuration and material properties custom optimized for various applications specifically. Moreover, the feature of batch manufacturing allows driving down the unit cost with a mature process flow in place.

Within imec / Holst Centre, surface micromachining technology has been developed to realize miniature thermopiles with polycrystalline silicon germanium (poly-SiGe). The distinct feature is that despite the use of surface micromachining technology, the thin film thermocouples are fabricated on top of a $6-\mu \mathrm{m}$ high topography so that the thermal resistance is greatly increased [16]. A scanning electron micrograph (SEM) photo of the fabricated thermocouples is given in Figure 9. One can clearly observe that the miniature thermocouple legs are suspended above the substrate thanks to the complete removal of sacrificial materials. In an individual thermopile chip, the total number of thermocouples varies between 1300 and 2500 . The total area of one chip measures about $3.0 \mathrm{~mm} \times 2.5$ $\mathrm{mm}$ while an area of about $1.0 \mathrm{~mm} \times 2.5 \mathrm{~mm}$ is occupied by the thermocouples. 


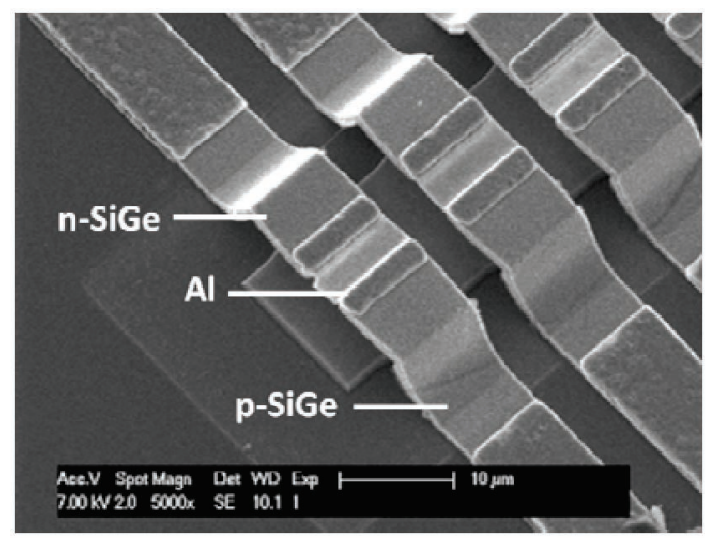

Figure 9. SEM photo of the poly-SiGe thermocouples fabricated on high topography [16].

After bonded to another Si chip on top, the fabricated thermopile is characterized under a variable temperature difference on a dedicated experimental setup. As shown in Figure 10, the open-circuit voltage output exceeds $1.2 \mathrm{~V}$ for a temperature difference of about $20^{\circ} \mathrm{C}$. On the other hand, the power output on a matched external load can still be substantially improved once the internal electrical resistance is optimized.

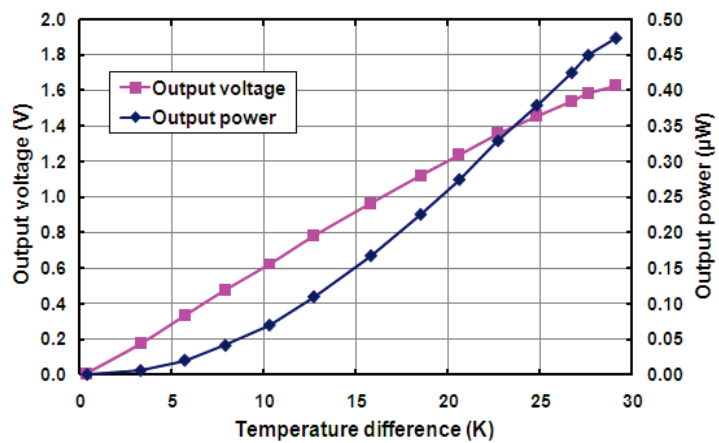

Figure 10. Voltage and power output of the fabricated thermopile under a variable temperature difference [16].

\section{CONCLUSION}

As the enabling technology for autonomous wireless sensor nodes, energy harvesting has attracted intensive interest worldwide. Cost-effective miniature energy harvesting devices can be approached by using micromachining technologies. This paper focuses on the principle and technical implementation of micromachined piezoelectric and thermoelectric harvesting devices. AlN-based piezoelectric devices are vacuum packaged by wafer level bonding to two glass wafers with etched cavities, resulting in a signficant gain in output performance. Further development is related to the improvement of package hermeticity. Consisting of a large number of poly-SiGe thermocouples, the micromachined thermopile delivers a voltage of about $1.2 \mathrm{~V}$ with a temperature difference of $20^{\circ} \mathrm{C}$. Its power output can be further increased upon further optimization of material properties.

\section{REFERENCES}

[1] S. Kamarudin, W. Daud, S. Ho, and U. Hasran. Overview on the challenges and developments of micro-direct methanol fuel cells (DMFC). J. Power Sources, vol. 163, pp. 743-754, 2007.

[2] B. W. Cook, S. Lanzisera, and K. S. J. Pister. SoC Issues for RF Smart Dust. Proc. IEEE 94, pp.11771196, 2006

[3] P. D. Mitcheson, E. M. Yeatman, G. K. Rao, A. S. Holmes, T. C. Green, Energy Harvesting From Human and MachineMotion for Wireless Electronic Devices Proceedings of the IEEE Vol. 96, pp. 1457-1486, 2008

[4] Tom Torfs, Vladimir Leonov, Chris Van Hoof, Bert Gyselinckx Body-Heat Powered Autonomous Pulse Oximeter. 5th IEEE Conference on Sensors pp. 427430.2006

[5] V. Pop, J. Penders, R. van Schaijk, R. Vullers, The limits and challenges for power optimization and system integration in state-of-the-art Wireless Autonomous Transducer Solutions, Proc. 3rd European Conf. Smart Systems Integration, Brussels, Belgium, March 10-11, 2009. pp. 544-547

[6] Vullers et al, Micropower Energy Harvesting, SolidState Electronics 53 (7) pp. 684-693, DOI: 10.1016/j.sse.2008.12.011.

[7] S. P. Beeby et al., Energy harvesting vibration sources for Microsystems applications, Meas. Sci. Technol. 17, R175, 2006

[8] N.S. Hudak and G.G. Amatucci, Small-scale energy harvesting through thermoelectric, vibration, and radiofrequency power conversion, J. Appl. Phys. 103, 101301, 2008

[9] C B Williams and R B Yates, Transducers '95, EuroSensors IX, the 8th International Conference on Solid State Sensors and Actuators, and European Sensors IX, Stockholm, Sweden, June 25 - 29, 1995

[10] P D Mitcheson, T C Green, A S Holmes, J. Microelectromechanical Sys., 13, 429, 2004.

[11] S Roundy, J. Intell. Mater. Sys. Struct., 809, 2005

[12] S Roundy, P K Wright, Smart Mater. Struct., 13, 1131, 2004.

[13] R. Elfrink et al., Vibration energy harvesting with aluminum nitride-based piezoelectric devices, J. Micromech. Microeng. 19, 094005, 2009

[14] M. Esashi, Wafer level packaging of MEMS, J. Micromech. Microeng. 18, 073001, 2008

[15] D. M. Rowe, editor. CRC Handbook of Thermoelectrics. CRC Press, Boca Raton, FL, Jul. 1995.

[16] J. Su, V. Leonov, M. Goedbloed, Y. van Andel, M. C. de Nooijer, R. Elfrink, Z. Wang and R. J. M. Vullers, Batch process micromachined thermoelectric energy harvester: fabrication and characterization, accepted to Journal of Micromechanics and Microengineering. 